\title{
Note sur la recherche et le dosage de l'acide pyruvique dans les liquides biologiques
}

\author{
por J. BALIS
}

L'acide pyruvique est le point d'aboutissement de la première étape du cycle de KREBS, pendant laquelle le glucose ou certains autres oses tels que le fructose sont dégradés sans intervention de l'oxygène.

C'est donc un témoin du catabolisme glucidique, et 1 est important de pouvoir le caractériser et le doser dans un milieu bıologıque.

Nous inspirant de la méthode de CARON et RAQUET, nous avons mis au point une technique permettant non seulement la mise en évidence de l'acide pyruvique mais également son dosage, spécialement dans les milieux liquides à base de sang, que nous utilisons pour les essais de culture de Trypanosoma evansi.

\section{Mise en évidence}

On utilise la coloration que donne l'acide pyruvique avec le nitroprussiate de sodium et l'ammoniaque en présence de sulfate d'ammonium à saturation.

Généralement, les liquides à examiner doivent subir une défécation que l'on réalise par l'emploi du sulfate d'ammonium à saturation.

Dans la majorité des cas, on obtient après filtration un liquide absolument incolore.

Avec les milieux peptonés ou le bouillon nutri†if, les résultats sont moins bons mais permettent, néanmoins de déceler l'acide pyruvique.

Nous avons adopté la technique suivante:

On ajoute à une prise de $5 \mathrm{ml}$, la quantité de sulfate d'ammonium nécessaire pour obtenir la saturation, soit 4 à 5 grammes.

Après une agitation de 3 à 4 mınutes, au bout de laquelle doit persister un peu de sulfate non dissous, on filtre sur papier.

Rev. Elev. Méd. vét. Pays trop. 1963, 16, no 4.

Reçu pour publication : novembre 1963.
A $2 \mathrm{ml}$ de liquide incolore recueilli, on ajoute dans l'ordre et en agitant après chaque opération :

- 4 gouttes de solution aqueuse récente de nitroprussiate de sodium à $10 \mathrm{p} .100$.

$-1 \mathrm{ml}$ d'ammoniaque officinal.

Si l'échantillon contient de l'acide pyruvique, on observe après quelques instants une coloration allant du vert clair au bleu foncé selon la concentration.

A $35^{\circ}$, la coloration atteint son maximum d'intensité en 6 à 8 minutes, reste relativement stable 10 à 12 minutes puis s'éclaircit progressivement. Ces valeurs sont à modifier en fonction de la température (voir tableau no 1).

TABLEAU No 1

\begin{tabular}{|c|c|c|}
\hline Température & $\begin{array}{c}\text { Lecture au } \\
\text { bout de } \\
\text { (en minutes) }\end{array}$ & $\begin{array}{c}\text { Durée de } \\
\text { stabilité } \\
\text { (en minutes) }\end{array}$ \\
\hline 150 & $\begin{array}{c}24 \text { à } 32 \\
12 \text { à } 16 \\
6 \text { à } 8\end{array}$ & $\begin{array}{c}40 \text { à } 48 \\
20 \text { à } 24 \\
10 \text { à } 12\end{array}$ \\
$35^{\circ}$ & \\
\hline
\end{tabular}

Spécificité de la réaction: Elle n'est pas absolue puisqu'elle se produit également 'avec l'acide acétone-carboxylique et l'acide oxaloocétique, or, ce dernier est justement le point de départ de la seconde phase du cycle de KREBS.

Cette réserve étant faite, la réaction conserve une grande partie de sa valeur car elle permet au moıns la mise en évidence d'une dégradation incomplète des glucides.

\section{Dosage}

Il est effectué en comparant la couleur obtenue, selon la technique que nous venons de décrire, à 
TABLEAU No 2

\begin{tabular}{|c|c|c|c|c|c|c|c|c|c|c|c|c|c|c|c|c|c|c|c|c|c|}
\hline Tube No & 1 & 2 & 3 & 4 & 5 & 6 & 7 & 8 & 9 & 10 & 11 & 12 & 13 & 14 & 15 & 16 & 17 & 18 & 19 & 20 & 21 \\
\hline Solution A (en ml). & 6 & 6,2 & 6,4 & 6,5 & 6,8 & 7 & 7,2 & 7,4 & 7,6 & 7,8 & 8 & 8,2 & 8,4 & 8,6 & 8,8 & 9 & 9,2 & 9,4 & 9,6 & 9,8 & 10 \\
\hline Solution $B(\mathrm{en} \mathrm{ml})$ & 4 & 3,8 & 3,6 & 3,4 & 3,2 & 3 & 2,8 & 2,6 & 2,4 & 2,2 & 2 & 1,8 & 1,6 & 1,4 & 1,2 & 1 & 0,8 & 0,6 & 0,4 & 0,2 & 0 \\
\hline $\begin{array}{l}\text { Concentration du } \\
\text { pyruvate (en } \mathrm{mg} \\
\text { par } \mathrm{ml} \text { ) } \ldots \ldots \ldots\end{array}$ & 0,4 & 0,38 & 0,36 & 0,34 & 0,32 & 0,3 & 0,28 & 0,26 & 0,24 & 0,22 & 0,20 & 0,18 & 0,16 & 0,14 & 0,12 & $0,1 \mid$ & 0,08 & 0,06 & 0,04 & 0,02 & 0 \\
\hline
\end{tabular}

celle d'une gamme étalon dont les concentrations vont de $0,02 \mathrm{mg}$ à $0,4 \mathrm{mg}$ de pyruvate de sodium par ml.

Cette gamme étalon est obtenue en mélangeant en proportions définies 2 solutions $A$ et $B$ (voir tableau $n^{0}$ 2) dont la composition est la suivante :

A: Sulfate d'ammonium à saturation dans l'eau distillée.

B : $100 \mathrm{ml}$ de solution $\mathrm{A}+100 \mathrm{mg}$ de pyruvate de sodium.

Cette gamme peutse conserver au moins 2 mois en flacons bouchés dans un endroit frais. Le 5ulfate d'ammonium à saturation empêche la culture des germes antotrophes qui feraient disparaître le pyruvate de sodium en l'espace de 48 heures.

\section{Sensibilité de la méthode}

La méthode n'est valable que pour les quantités d'acide pyruvique allant de $0,02 \mathrm{mg}$ par $\mathrm{ml}$ à $0,4 \mathrm{mg}$ par $\mathrm{ml}$.

En effet, on n'obtient une teinte verdâtre tranchant nettement avec celle du tube témoin sans pyruvate qu'à partir de $0,02 \mathrm{mg}$ par $\mathrm{ml}$ ce qui correspond à une solution à 1 pour 50.000

Par contre pour une concentration supérieure. à 1 pour 2.500 sout $0,4 \mathrm{mg}$ par $\mathrm{ml}$ ił est difficile de faire une évaluation, la coloratıon bleue étant trop intense.

\section{Conclusion}

La coloration que donne l'acide pyruvique avec le nitroprussiate de sodium et l'ammoniaque en présence de sulfate d'ammonium à saturation, permet une recherche et un dosage relativement faciles.

Comple tenu des réserves formulées à propos de la spécificité, cette méthode permet tout au moins de mettre en évidence une dégradation incomplète des glucides dans un milieu biologique.

- Laboratore de recherches vétérinaire de Farcha - Fort-Lamy (Rép. du Tchad).

- Service d'entomo-protozoologie.

\section{BIBLIOGRAPHIE}

CARON (H.) et RAQUET (D.). - Caractérisation et dosage de l'acide pyruvique. Application à la recherche de l'acide lactique. J. pharm. Chim (Poris), 1942, A. 2: 333.

Note présentée à la sécnce du 5 novembre 1941 de la Société de Pharmacie de Parıs.

\section{SUMMARY}

\section{A note on research and the dosage of pyruvic acid in biological liquids.}

The colouration given by pyruvic acid by the nitroprussiate of sodium and ammonia in the presence of a saturated solution of ammonium sulphate permits its detection and in relatively low titres.

Bearing in mind reservations regarding specificity, this method gives evidence to some degree of incomplete dissipation of glucides in a biological medium. 


\section{RESUMEN}

Nota sobre la infestigacion y el dosaje del acido piruvico en los liquidos biologicos.

La coloracion que da el acido piruvico con el nitroprusiato de sodio y el amoniaco en presencia de sulfato de amonio a saturacion, permite una investigacion y un dosaje relativamente faciles.

Teniendo en cuenta las reservas formuladas a proposito de la especificidad, este métodopermite al menos poner en evidencia una degradacion incompleta de los glucidos en un medio biologico. 